\title{
Determinación de intervalos biológicos de referencia para adultos en el equipo hematológico BC-5000 de la Escuela de Microbiología de la Universidad de Antioquia, Medellín 2017
}

\author{
Simón Santiago Mejía-Saldarriaga' , Daniela Agudelo Rendón 2 , Felipe Bossio-Zapata ${ }^{3}$, Érika Sánchez-Cifuentes4, \\ Luz Marina Jaramillo-Pérez ${ }^{5}$, Paola Andrea Acevedo-Toro ${ }^{6}$
}

\section{RESUMEN}

Introducción: los valores del hemograma en adultos pueden variar de acuerdo con factores como el origen étnico, la edad, el sexo y la altura sobre el nivel del mar, por lo cual es indispensable conocer intervalos biológicos asociados a nuestra población. Por esto el objetivo de nuestro estudio fue determinar intervalos biológicos de referencia (IBR) para adultos en un equipo hematológico BC-5000.

Metodología: estudio descriptivo transversal con 111 donantes entre 18 y 62 años de un banco de sangre de Medellín. Se realizó un hemograma, proteína $C$ reactiva y ferritina. Se calcularon Ios IBR siguiendo las recomendaciones de la Federación Internacional de Química Clínica. La comparación de los IBR según sexo se realizó con la prueba T-student.

Resultados: Ios IBR calculados mediante la fórmula $\mathrm{X} \pm(\mathrm{DS} * 1,96)$ mostraron diferencias estadísticamente significativas entre hombres y mujeres para el recuento de la mayoría de los parámetros. Se obtuvieron medias estadísticas mayores en mujeres para el recuento de leucocitos y plaquetas, recuentos absolutos y relativos de alounas células blancas, volumen plaquetario medio (VPM) y plaquetocrito (PCT).

1 Microbiólogo y Bioanalista, Universidad de Antioquia, Medellín, Colombia.

Microbióloga y Bioanalista, Universidad de Antioquia, Medellín, Colombia.

Estudiante de pregrado, Escuela de Microbiología, Universidad de Antioquia, Medellín, Colombia

4 Microbióloga y Bioanalista, servicio social obligatorio, Universidad de Antioquia, Medellín, Colombia.

5 Bacterióloga y Laboratorista Clínica, Esp, MSc. Docente, coordinadora de laboratorio docente de hematología, Escuela de Microbiología, Universidad de Antioquia, Medellín, Colombia.

6 Microbióloga y Bioanalista, MS en ciencias básicas biomédicas. Coordinadora grupo Hematopatología Molecular, Docente Escuela de Microbiología, Universidad de Antioquia, Medellín, Colombia.

Correspondencia: Paola Andrea Acevedo-Toro; paola.acevedo@udea.edu.co

Recibido: junio 02 de 2018

Aceptado: septiembre 13 de 2018

Cómo citar: Mejía-Saldarriaga SS, Agudelo Rendón D, Bossio-Zapata F, Sánchez-Cifuentes E, Jaramillo-Pérez LM, Acevedo-Toro P. Determinación de intervalos biológicos de referencia para adultos en el equipo hematológico BC-5000 de la Escuela de Microbiología de la Universidad de Antioquia, Medellín 2017. latreia. 2019 Abr-Jun; 32(2):92-101. D0I 10.17533/udea.iatreia.08. 
Discusión: la presencia de andrógenos en el sexo masculino, inductores del sistema eritropoyético, así como los estrógenos y el período menstrual, supresores del mismo, pueden explicar los valores menores en el recuento eritrocitario, en los rangos de hemoglobina, hematocrito y glóbulos rojos en las mujeres. Las altas cargas de estrógenos e interleucinas 6 y 10 en las mujeres amplían la vida media de células como linfocitos, explicando la diferencia en el recuento leucocitario a expensas de linfocitos encontrados en el presente estudio.

\section{PALABRAS CLAVE}

Biometría; Donantes de Sangre; Hematología; Intervalos de Referencia; Parámetros

\section{SUMMARY}

Determination of reference biological intervals in a hematology analyzer BC-5000 of the Microbiology School of the University of Antioquia, Medellin 2017

Introduction: The adult hemogram values can vary according factors such as ethnic origin, age, sex and level above the sea, whereby it is essential to know the biological intervals associated to our own population. Therefore, the objective of our study was to determine biological reference intervals (BRI) for adults with the hematological equipment BC-5000.

Methods: This is a transversal descriptive study with 111 blood bank donors from Medellín, between 1862 years old. Hemogram, $C$ reactive protein and ferritin were done. The BRI were calculated according to the recommendations of the International Federation of Clinical Chemistry (IFCC), with the formula X \pm (DS * $1,96)$. BRI comparison according sex was carried out with the t-student test.

Results: Calculated BRI by $\mathrm{X} \pm(\mathrm{DS} * 1,96)$ formula, showed statistically significant differences $(P<0,05)$ between men and women for count in most of the parameters. Higher statistical means were obtain in women for the count of leukocytes and platelets, absolute counts of some white cells, VPN y PCT.

Discussion: The presence of androgens in the male sex, inductors of the erythropoiesis system, as well as the estrogens and menstrual period, suppressor of this system, can explain the lower values in the female erythrocyte count, the hemoglobin, hematocrit and red cell lower ranges. The hiog estrogen and interleukins 6 and 1 loads in women, drive the extended half-life of some cells like lymphocytes, that can explain the difference in leucocyte count found in the present study.

\section{KEY WORDS}

Blood Donors; Biometrics; Hematology; Parameters; Reference Intervals

\section{INTRODUCCIÓN}

El hemograma es una de las pruebas más solicitadas en el laboratorio clínico. Se constituye en un examen de apoyo diagnóstico y de gran utilidad, que no solo se restringe a las enfermedades hematológicas sino también como una herramienta de estudio que aporta mayor información al médico sobre la homeostasis de un individuo, al proveer un análisis cuantitativo y cualitativo de las células sanguíneas y sus componentes (1-3).

A través del tiempo, el hemograma ha sido modificado de acuerdo con el desarrollo tecnológico, Ios parámetros que lo componen, la forma de interpretarlos y los recursos con que cuenta el laboratorio, aumentando el número de parámetros sanguíneos medibles por un equipo y automatizando el procesado de muestras y producción de resultados $(1,3)$. El equipo hematoIógico Mindray BC-5000 ${ }^{\circledR}$ usa los siguientes métodos de medición: impedancia eléctrica para determinar los datos de los glóbulos rojos (RBC por sus siglas en inglés) y plaquetas (PLT por sus siglas en inglés); el método colorimétrico para determinar la hemoglobina (HGB por sus siglas en inglés) y dispersión por láser para determinar los datos de glóbulos blancos (WBC por sus siglas en inglés).

Algunos parámetros se obtienen directamente como los anteriores y otros mediante cálculos. Finalmente, se obtienen 23 parámetros en una muestra de sangre total (4). Estos parámetros se agrupan en eritrograma, leucograma y plaquetograma. El eritrograma está compuesto por el recuento de eritrocitos, la concentración de hemoglobina, hematocrito, los índices 
eritrocitarios (volumen corpuscular medio, hemoglobina corpuscular media, concentración de hemoglobina corpuscular media) y el ancho de distribución eritroide. El leucograma comprende el recuento total, absoluto y relativo de leucocitos y el plaquetograma incluye el recuento de plaquetas, el volumen medio de plaquetas, el ancho de distribución plaquetario y el plaquetocrito $(1,4)$.

Para la correcta interpretación del hemograma y de sus parámetros, es necesario establecer los valores de decisión clínica o intervalos biológicos de referencia (IBR), acordes con la población y propios de la tecnología utilizada (5). Los IBR están definidos como los intervalos donde se sitúan el $95 \%$ de los valores medidos en una población definida $(6,7)$. El Instituto de Estándares Clínicos y de Laboratorio (Clinical and Laboratory Standards Institute, CLSI) (8), la Federación Internacional de Química Clínica (IFCC) (9) y el Instituto Colombiano de Normas Técnicas y Certificación (ICONTEC) (6), desarrollaron distintas guías donde proponen, definen y estipulan metodologías para el cálculo de los IBR en el laboratorio clínico; sin embargo, la determinación de estos es competencia de los laboratorios y se deben establecer en función de la población a la que presta sus servicios (9).

Además, para la interpretación de Ios IBR es indispensable conocer los factores asociados capaces de introducir variaciones en cada parámetro del hemograma (10). Para ello se deben definir las condiciones de salud de los individuos participantes y los indicadores biológicos que permiten identificarlos como sanos (11). Algunas de las pruebas que aportan a esta caracterización son la proteína $C$ reactiva $(\mathrm{PCR})$ y la ferritina.

La PCR es una proteína sintetizada en el hígado, caracterizada por encontrarse circulando en sangre periférica como respuesta a un suceso inflamatorio, por lo que ha sido de gran ayuda en el diagnóstico y análisis de enfermedades infecciosas e inflamatorias (12). La ferritina es una proteína intracelular secretada al plasma en pequeñas cantidades que puede almacenar hasta 4500 átomos de hierro. La medición de esta proteína evidencia el estado de los depósitos de hierro; sin embargo, su utilidad va más allá pues investigaciones realizadas por Coy et al. demostraron que una disminución en los valores de ferritina podría indicar procesos inflamatorios $y / 0$ infecciosos, debido a que las citoquinas liberadas desencadenan vías de inflamación que inhiben la absorción de hierro en el intestino y no dejan liberarlo por el sistema mononuclear fagocítico, disminuyendo la disponibilidad de hierro (13).

Entre los parámetros fisiológicos reportados en la literatura que inducen una mayor variación en el hemograma, están: el origen étnico, la edad, el sexo y la altura sobre nivel del mar de la ciudad de residencia (13-15). No obstante, muy pocas instituciones o laboratorios determinan sus IBR e incluyen como referencia los reportados en trabajos de investigación y/o instructivos comerciales (5). Asimismo, la mayoría de los artículos revisados no ahondan en la descripción de la población de referencia, en el procedimiento de medición ni en los instrumentos usados, por lo cual se debe insistir en que cada laboratorio clínico defina sus respectivos IBR de acuerdo con la población, la instrumentación y los factores ambientales que puedan modificar los parámetros del hemograma al comprometer la exactitud en la medición y, por lo tanto, la confiabilidad de los resultados.

EI ICONTEC recomienda, en la guía de requisitos generales para la calidad y la competencia de laboratorios clínicos (NTC-ISO 15189), revisar, y de ser el caso, recalcular Ios IBR cuando se cambien los equipos, procedimientos analíticos o preanalíticos, cuando ya no sean pertinentes para la población objeto del estudio y cuando el personal encargado del laboratorio lo considere necesario $(6,9)$.

Investigaciones anteriores sugieren un aumento en el recuento absoluto de neutrófilos en condiciones de estrés o ejercicio intenso por el paso de estas células del pool circulante hacia el marginal; asimismo, las venopunciones complicadas y la agitación excesiva de la muestra causan pseudotrombocitopenias como consecuencia de una activación plaquetaria, formando microcoágulos (16). Además, se ha planteado diferencias en el recuento absoluto de neutrófilos según la edad y la población étnica a la cual se pertenezca (17).

No solo se deben tener en cuenta las variaciones fisiológicas y ambientales para una mejor determinación del IBR y una emisión de resultados confiables por parte del equipo hematológico, sino también asegurar que el instrumento y los procedimientos de medida utilizados tengan un desempeño analítico 
aceptable; es decir, que este sea adecuado para su uso y cumpla con las especificaciones del fabricante. Por esto, antes de emitir resultados, es aconsejable realizar la verificación del equipo de acuerdo con los requisitos establecidos por el fabricante y las normas de calidad correspondientes (18).

En este orden de ideas, este trabajo pretende determinar los IBR para adultos en el equipo hematológico BC-5000 del laboratorio de docencia de la Escuela de Microbiología de la Universidad de Antioquia, en una población de individuos sanos que son donantes no repetitivos del Banco de Sangre de la Escuela de Microbiología, con el fin de dar importancia, incentivar y mejorar los análisis de los parámetros hematológicos, brindando seguridad y confiabilidad en la interpretación del hemograma.

\section{METODOLOGÍA}

Estudio descriptivo transversal en el que se captaron 119 donantes del banco de sangre de la Escuela de Microbiología de la Universidad de Antioquia (U de A), seleccionados consecutivamente entre el mes de agosto y septiembre de 2017. Para cada donante se recolectaron 2 muestras de sangre, un tubo tapa lila con anticoagulante EDTA para realizar el hemograma y un tubo seco tapa roja para realizar las pruebas de ferritina y PCR. Los donantes cumplieron con Ios siguientes criterios de inclusión: ser mayor de 18 y menor de 62 años, residente de la ciudad de Medellín y no ser donante repetitivo; además de cumplir con Ios requisitos de selección del banco de sangre según lo estipulado en el decreto 1571 de 1993 del Ministerio de la Protección Social (19). EI tamaño muestral dependió de la cantidad de las pruebas de ferritina y PCR (proteína $C$ reactiva) que fueron financiadas, y que se establecieron como necesarias para disminuir al máximo los factores asociados que pudieran inducir variaciones en los resultados, lo que constituyó una clara limitación al momento de establecer la cantidad de muestras captadas.

Se excluyeron los donantes que obtuvieron resultados de ferritina y PCR anormales. Se establecieron Ios valores normales de ferritina entre 10 y $291 \mathrm{ng} / \mathrm{ml}$ en mujeres, $y$ de 22 a $322 \mathrm{ng} / \mathrm{ml}$ en hombres. La ferritina se midió por la técnica de quimioluminiscencia
(ADVIA Centaur ${ }^{\circledR}$ XP System). Para la PCR se consideraron como resultados normales de 0 a $1 \mathrm{mg} / \mathrm{dL}$. La PCR se determinó por inmunoturbidimetría (ADVIA ${ }^{\circledR}$ 1800 Clinical Chemistry System) Los valores de referencia de la PCR y ferritina se tomaron de los insertos del fabricante de los reactivos. Ambas pruebas se procesaron en el laboratorio clínico de la Clínica León XIII de Medellín. Se excluyeron 4 participantes por ferritina fuera de lo normal y 4 con la prueba de PCR alterada, para un total de 111 donantes.

Los hemogramas fueron procesados en el equipo Mindray $\mathrm{BC}-5000^{\circledR}$ del laboratorio de docencia de la Escuela de Microbiología. El equipo hematológico fue verificado según la guía EP15-A3 VERIFICACIÓN DE LA PRECESIÓN Y ESTIMACIÓN DEL SESGO deI CLSI, cumpliendo con las especificaciones del fabricante para los parámetros evaluados (20).

Para el análisis de los parámetros del hemograma se eliminaron valores atípicos con métodos gráficos y estadísticos: caja de bigotes y estadística de Grubbs, respectivamente, para evitar desproporcionar los resultados $y$ asegurar una interpretación correcta. La descripción se hizo a través de medidas de tendencia central (media, mediana) y dispersión (desviación estándar y rangos intercuartílicos); la variable sexo se describió a través de frecuencias absolutas y relativas.

Se calcularon los IBR para cada parámetro del hemograma con base en la investigación de Molina et al, quienes utilizaron los intervalos de confianza del $95 \%$ para la media a través de la fórmula $\mathrm{X} \pm \mathrm{Zalfa} / 2$ * $\mathrm{DS} / \sqrt{ } \mathrm{n}$ (Tabla 1) (5). Adicionalmente, se tuvieron en cuenta los lineamientos de la IFCC, la cual establece la fórmula, media más o menos desviación estándar de cada parámetro por 1,96 que es una constante (X \pm [DS * 1,96]), para determinar eI IBR (9). La comparación de Ios IBR según el sexo se realizó con la prueba T-student, ya que se cruzaron variables cuantitativas (parámetros del hemograma) con una cualitativa dicotómica (sexo), verificando previamente, el supuesto de normalidad con el estadístico de Shapiro Wilk, evaluada por Isaza et al., en muestras poblacionales similares a las usadas en esta investigación, reportando un desempeño adecuado (21). Todos los análisis se realizaron en SPSS versión 24 y Epidat versión 3.1, se consideraron valores $P<0,05$ como valores significativos. 
Tabla 1. Intervalos biológicos de referencia del hemograma por sexo a través de intervalos de confianza del $95 \%$ para la media $\left(X \pm Z_{\text {alfa/2 }} * D S / \sqrt{ } n\right)$

\begin{tabular}{|c|c|c|c|c|}
\hline \multirow[b]{2}{*}{ Parámetro } & \multicolumn{2}{|c|}{ Femenino } & \multicolumn{2}{|c|}{ Masculino } \\
\hline & Media \pm DS & IBR & Media \pm DS & IBR \\
\hline WBC $\left(10^{3} / \mu \mathrm{L}\right)$ & $7,45 \pm 1,33$ & $7,13-7,77$ & $6,36 \pm 1,23$ & $5,99-6,73$ \\
\hline NEU\# $\left(10^{3} / \mu \mathrm{L}\right)$ & $4,20 \pm 1,00$ & $3,95-4,45$ & $3,50 \pm 0,88$ & $3,23-3,77$ \\
\hline LYM\# $\left(10^{3} / \mu \mathrm{L}\right)$ & $2,58 \pm 0,54$ & $2,45-2,71$ & $2,21 \pm 0,51$ & $2,05-2,37$ \\
\hline MON\# $\left(10^{3} / \mu \mathrm{L}\right)$ & $0,52 \pm 0,13$ & $0,48-0,54$ & $0,49 \pm 0,12$ & $0,45-0,52$ \\
\hline EOS\# $\left(10^{3} / \mu \mathrm{L}\right)$ & $0,10 \pm 0,06$ & $0,09-0,12$ & $0,09 \pm 0,04$ & $0,07-0,1$ \\
\hline BAS\# $\left(10^{3} / \mu \mathrm{L}\right)$ & $0,00 \pm 0,01$ & $0,00-0,00$ & $0,00 \pm 0,00$ & $0,00-0,00$ \\
\hline NEU (\%) & $56,48 \pm 6,83$ & $54,82-58,15$ & $55,38 \pm 7,17$ & $53,19-57,55$ \\
\hline LYM (\%) & $34,22 \pm 6,04$ & $32,75-35,69$ & $34,77 \pm 6,96$ & $32,65-36,88$ \\
\hline MON (\%) & $6,91 \pm 1,54$ & $6,53-7,29$ & $7,65 \pm 1,41$ & $7,22-8,08$ \\
\hline EOS (\%) & $1,61 \pm 1,35$ & $1,28-1,93$ & $1,38 \pm 0,69$ & $1,17-1,59$ \\
\hline BAS (\%) & $0,07 \pm 0,10$ & $0,04-0,09$ & $0,05 \pm 0,08$ & $0,03-0,07$ \\
\hline $\mathrm{RBC}\left(10^{6} / \mu \mathrm{L}\right)$ & $4,57 \pm 0,31$ & $4,49-4,65$ & $5,15 \pm 0,30$ & $5,06-5,24$ \\
\hline HGB (g/dL) & $13,80 \pm 0,81$ & $13,6-14,00$ & $15,93 \pm 0,96$ & $15,68-16,18$ \\
\hline HCT (\%) & & $39,79-40,85$ & $45,90 \pm 2,59$ & $45,11-45,90$ \\
\hline $\mathrm{MCV}(\mathrm{fL})$ & $88,47 \pm 3,93$ & $87,64-89,30$ & $87,71 \pm 3,44$ & $86,66-88,76$ \\
\hline $\mathrm{MCH}(\mathrm{pg})$ & $30,23 \pm 1,41$ & $29,89-30,57$ & $30,34 \pm 1,29$ & $29,95-30,73$ \\
\hline $\mathrm{MCHC}(\mathrm{g} / \mathrm{dL})$ & $34,26 \pm 0,63$ & $34,11-34,41$ & $34,69 \pm 0,69$ & $34,48-34,90$ \\
\hline RDW-CV (\%) & $12,83 \pm 0,70$ & $12,70-12,96$ & $12,63 \pm 0,54$ & $12,47-12,79$ \\
\hline RDW-SD (fL) & $43,93 \pm 2,24$ & $43,38-44,48$ & $43,39 \pm 2,01$ & $42,78-44,00$ \\
\hline $\operatorname{PLT}\left(10^{3} / \mu \mathrm{L}\right)$ & $273,39 \pm 50,35$ & $261,11-285,67$ & $250,86 \pm 44,19$ & $237,43-264,30$ \\
\hline VMP (fL) & $10,35 \pm 0,98$ & $10,11-10,59$ & $9,90 \pm 1,02$ & $9,59-10,21$ \\
\hline PDW & $15,80 \pm 0,27$ & $15,73-15,87$ & $15,89 \pm 0,31$ & $15,80-15,98$ \\
\hline PCT (\%) & $0,28 \pm 0,04$ & $0,27-0,29$ & $0,25 \pm 0,04$ & $0,23-0,26$ \\
\hline
\end{tabular}

WBC: recuento total de leucocitos, NEU: neutrófilos, LYM: linfocitos, MON: monocitos, EOS: eosinófilos, BAS: basófilos, \#: recuento absoluto, \%: recuento relativo, RBC: recuento de eritrocitos, HGB: hemoglobina, HCT: hematocrito, MCV: volumen corpuscular medio, $\mathbf{M C H}$ : hemoglobina corpuscular media, MCHC: concentración de hemoglobina corpuscular media, RDW: ancho de distribución eritroide, PLT: recuento de plaquetas, VMP: volumen plaquetario medio, PDW: ancho de distribución plaquetario, PCT: plaquetocrito. Tomado de: Molina K, Vargas E, Tavera S, Pérez R, Mantilla Y, Cardona J. Intervalos biológicos de referencia del hemograma en personas sanas, Medellín, 2012. Medicina \& Laboratorio. 2013;19(5-6):267-81.

\section{CONSIDERACIONES ÉTICAS}

Cada individuo participó voluntariamente y se obtuvo su consentimiento informado por escrito antes del estudio. Esta investigación fue sometida y posteriormente aprobada por el Comité de Ética de la Escuela de Microbiología de la Universidad de Antioquia. Igualmente, se realizó bajo el marco de los principios éticos en salud expuestos en la resolución 8430 de 1993, establecida por el Ministerio de salud de la República de Colombia (22). Los riesgos para la realización de la investigación fueron mínimos, no generaron incapacidades temporales o permanentes a los individuos. Además, no hubo compromiso psicológico, legal, social, entre otros.

\section{RESULTADOS}

La población femenina representó el 61,1 \% (n = 111) y el $50 \%$ de los participantes tenían entre 20 y 30 años, con una media de 26 años ( \pm 9 DS), la edad mínima fue de 18 años y la máxima de 55. Se obturo una media de $7,01 \times 10^{3} / \mu \mathrm{L}$ para el recuento de 
leucocitos, $4,79 \times 10^{6} / \mu \mathrm{L}$ para el recuento de eritrocitos, $264,38 \times 10^{3} / \mu \mathrm{L}$ para el recuento de plaquetas $y$ 14,63 g/dL para la concentración de hemoglobina, además de una media de 42,49 para el hematocrito (Tabla 2).

Los IBR calculados mediante la fórmula $\mathrm{X} \pm$ (DS * 1,96) mostraron diferencias estadísticamente significativas $(P<0,05)$ entre hombres 8 mujeres para los parámetros de recuento de leucocitos 8 eritrocitos, recuento absoluto de neutrófilos y linfocitos, porcentaje relativo de monocitos, hemoglobina, hematocrito, MCHC, VMP, PDW y PCT. Se obtuvieron medias estadísticas mayores en mujeres para el recuento de leucocitos y plaquetas, recuentos absolutos de neutrófilos, linfocitos, monocitos, eosinófilos, porcentaje relativo de neutrófilos, eosinófilos y basófilos, VPM y PCT, a excepción del recuento absoluto de basófilos, el cual fue igual tanto en hombres como en mujeres (Tabla 3). Estas diferencias confirman la importancia de la variable sexo en el cálculo de los IBR del hemograma (23).

Tabla 2. Características demográficas y hematológicas de la población de estudio

\begin{tabular}{|c|c|c|c|}
\hline Variable & Media \pm DS & Mediana (RIQ) & Mínimo - Máximo \\
\hline Edad & $26 \pm 9$ & $23(20-30)$ & $18-55$ \\
\hline WBC $\left(10^{3} / \mu \mathrm{L}\right)$ & $7,01 \pm 1,39$ & $6,82(6,03-7,93)$ & $4,16-10,57$ \\
\hline NEU\# $\left(10^{3} / \mu \mathrm{L}\right)$ & $3,92 \pm 1,01$ & $3,85(3,16-4,56)$ & $1,80-6,28$ \\
\hline LYM\# $\left(10^{3} / \mu \mathrm{L}\right)$ & $2,43 \pm 0,56$ & $2,41(2,07-2,78)$ & $1,26-3,86$ \\
\hline MON\# $\left(10^{3} / \mu \mathrm{L}\right)$ & $0,51 \pm 0,12$ & $0,50(0,41-0,58)$ & $0,26-0,83$ \\
\hline EOS\# $\left(10^{3} / \mu \mathrm{L}\right)$ & $0,10 \pm 0,06$ & $0,09(0,06-0,12)$ & $0,02-0,35$ \\
\hline BAS\# $\left(10^{3} / \mu \mathrm{L}\right)$ & $0,00 \pm 0,01$ & $0,00(0,00-0,01)$ & $0,00-0,02$ \\
\hline NEU (\%) & $56,06 \pm 6,95$ & $55,60(51,60-60,40)$ & $39,40-72$ \\
\hline LYM (\%) & $34,43 \pm 6,39$ & $34,35(29,85-38,65)$ & $16,90-49,40$ \\
\hline MON (\%) & $7,21 \pm 1,53$ & $7,00(6,10-8,20)$ & $3,90-11,10$ \\
\hline EOS (\%) & $1,52 \pm 1,14$ & $1,30(0,80-1,90)$ & $0,30-8,90$ \\
\hline BAS (\%) & $0,06 \pm 0,09$ & $0,00(0,00-0,10)$ & $0,00-0,40$ \\
\hline $\operatorname{RBC}\left(10^{6} / \mu \mathrm{L}\right)$ & $4,79 \pm 0,42$ & $4,84(4,51-5,05)$ & $3,88-5,60$ \\
\hline HGB (g/dL) & $14,63 \pm 1,35$ & $14,40(13,50-15,60)$ & $12,10-18,10$ \\
\hline HCT (\%) & $42,49 \pm 3,59$ & $42,00(39,60-44,8)$ & $35,30-51,70$ \\
\hline MCV (fL) & $88,16 \pm 3,41$ & $87,70(86,00-90,70)$ & $80,20-96,90$ \\
\hline $\mathrm{MCH}(\mathrm{pg})$ & $30,27 \pm 1,36$ & $30,3(29,40-31,20)$ & $25,30-33,10$ \\
\hline $\mathrm{MCHC}(\mathrm{g} / \mathrm{dL})$ & $34,43 \pm 0,69$ & $34,4(34,00-35,00)$ & $33,10-36,10$ \\
\hline RDW-CV (\%) & $12,75 \pm 0,65$ & $12,7(12,30-13,20)$ & $11,20-14,60$ \\
\hline RDW-SD (fL) & $43,71 \pm 2,16$ & $43,7(42,10-44,95)$ & $38,8-48,60$ \\
\hline $\operatorname{PLT}\left(10^{3} / \mu \mathrm{L}\right)$ & $264,38 \pm 49,04$ & $265(228,00-293,00)$ & $150,00-376,00$ \\
\hline VMP (fL) & $10,18 \pm 1,02$ & $10,20(9,50-10,90)$ & $8,10-12,30$ \\
\hline PDW & $15,87 \pm 0,30$ & $15,80(15,60-16,10)$ & $15,20-16,60$ \\
\hline РCT (\%) & $0,26 \pm 0,04$ & $0,26(0,24-0,29)$ & $0,17-0,36$ \\
\hline
\end{tabular}

WBC: recuento total de leucocitos, NEU: neutrófilos, LYM: linfocitos, MON: monocitos, EOS: eosinófilos, BAS: basófilos, \#: recuento absoluto, \%: recuento relativo, RBC: recuento de eritrocitos, HGB: hemoglobina, HCT: hematocrito, MCV: volumen corpuscular medio, $\mathbf{M C H}$ : hemoglobina corpuscular media, MCHC: concentración de hemoglobina corpuscular media, RDW: ancho de distribución eritroide, PLT: recuento de plaquetas, VMP: volumen plaquetario medio, PDW: ancho de distribución plaquetario, PCT: plaquetocrito. 
Tabla 3. Intervalos biológicos de referencia del hemograma por sexo $(X \pm(D S * 1,96))$

\begin{tabular}{|c|c|c|c|c|c|}
\hline \multirow[b]{2}{*}{ Parámetro } & \multicolumn{2}{|c|}{ Femenino } & \multicolumn{2}{|c|}{ Masculino } & \multirow{2}{*}{ Valor $P$} \\
\hline & Media \pm DS & IBR & Media \pm DS & IBR & \\
\hline WBC $\left(10^{3} / \mu \mathrm{L}\right)$ & $7,5 \pm 1,3$ & $4,85-10,1$ & $6,36 \pm 1,2$ & $3,95-8,8$ & $0,001^{*}$ \\
\hline NEU\# $\left(10^{3} / \mu \mathrm{L}\right)$ & $4,2 \pm 1,00$ & $2,23-6,2$ & $3,50 \pm 0,9$ & $1,77-5,2$ & $0,001^{*}$ \\
\hline LYM\# $\left(10^{3} / \mu \mathrm{L}\right)$ & $2,6 \pm 0,5$ & $1,52-3,6$ & $2,21 \pm 0,5$ & $1,21-3,2$ & $0,001^{*}$ \\
\hline MON\# $\left(10^{3} / \mu \mathrm{L}\right)$ & $0,52 \pm 0,1$ & $0,26-0,8$ & $0,49 \pm 0,1$ & $0,26-0,7$ & 0,27 \\
\hline EOS\# $\left(10^{3} / \mu \mathrm{L}\right)$ & $0,1 \pm 0,1$ & $0,00-0,2$ & $0,09 \pm 0,0$ & $0,00-0,2$ & 0,158 \\
\hline BAS\# $\left(10^{3} / \mu \mathrm{L}\right)$ & $0,00 \pm 0,01$ & $0,00-0,0$ & $0,00 \pm 0,0$ & $0,00-0,1$ & $0,022^{*}$ \\
\hline NEU (\%) & $56,5 \pm 6,8$ & $43,09-69,9$ & $55,38 \pm 7,2$ & $41,33-69,4$ & 0,422 \\
\hline LYM (\%) & $34,22 \pm 6,0$ & $22,39-46,1$ & $34,77 \pm 6,9$ & $21,12-48,4$ & 0,668 \\
\hline MON (\%) & $6,91 \pm 1,5$ & $3,88-9,9$ & $7,65 \pm 1,4$ & $4,89-10,4$ & $0,012^{*}$ \\
\hline EOS (\%) & $1,61 \pm 1,4$ & $0,00-4,3$ & $1,38 \pm 0,7$ & $0,04-2,7$ & 0,343 \\
\hline BAS (\%) & $0,07 \pm 0,1$ & $0,00-0,3$ & $0,05 \pm 0,1$ & $0,00-0,2$ & 0,23 \\
\hline $\mathrm{RBC}\left(10^{6} / \mu \mathrm{L}\right)$ & $4,57 \pm 0,3$ & $3,96-5,2$ & $5,15 \pm 0,3$ & $4,57-5,7$ & $0,001 *$ \\
\hline $\mathrm{HGB}(\mathrm{g} / \mathrm{dL})$ & $13,80 \pm 0,81$ & $12,22-15,38$ & $15,93 \pm 0,96$ & $14,05-17,80$ & $0,001^{*}$ \\
\hline HCT (\%) & $40,32 \pm 2,17$ & $36,07-44,56$ & $45,90 \pm 2,59$ & $40,83-50,97$ & $0,001^{*}$ \\
\hline $\mathrm{MCV}(\mathrm{fL})$ & $88,47 \pm 3,93$ & $81,83-95,10$ & $87,71 \pm 3,44$ & $80,97-94,46$ & 0,442 \\
\hline $\mathrm{MCH}(\mathrm{pg})$ & $30,23 \pm 1,41$ & $27,46-32,99$ & $30,34 \pm 1,29$ & $27,82-32,87$ & 0,665 \\
\hline $\mathrm{MCHC}(\mathrm{g} / \mathrm{dL})$ & $34,26 \pm 0,63$ & $33,02-35,49$ & $34,69 \pm 0,69$ & $33,33-36,05$ & $0,001^{*}$ \\
\hline RDW-CV (\%) & $12,83 \pm 0,70$ & $11,45-14,21$ & $12,63 \pm 0,54$ & $11,58-13,69$ & 0,119 \\
\hline RDW-SD (fL) & $43,93 \pm 2,24$ & $39,54-48,32$ & $43,39 \pm 2,01$ & $39,45-47,32$ & 0,206 \\
\hline $\operatorname{PLT}\left(10^{3} / \mu \mathrm{L}\right)$ & $273,39 \pm 50,35$ & $174,70-372,09$ & $250,86 \pm 44,19$ & $164,26-337,47$ & 0,338 \\
\hline VMP (fL) & $10,35 \pm 0,98$ & $8,43-12,28$ & $9,90 \pm 1,02$ & $7,90-11,90$ & $0,022^{*}$ \\
\hline PDW & $15,80 \pm 0,27$ & $15,27-16,32$ & $15,89 \pm 0,31$ & $15,37-16,58$ & $0,002^{*}$ \\
\hline РCT (\%) & $0,28 \pm 0,04$ & $0,20-0,35$ & $0,25 \pm 0,04$ & $0,17-0,32$ & $0,001 *$ \\
\hline
\end{tabular}

WBC: recuento total de leucocitos, NEU: neutrófilos, LYM: linfocitos, MON: monocitos, EOS: eosinófilos, BAS: basófilos, \#: recuento absoluto, \%: recuento relativo, RBC: recuento de eritrocitos, HGB: hemoglobina, HCT: hematocrito, MCV: volumen corpuscular medio, $\mathbf{M C H}$ : hemoglobina corpuscular media, MCHC: concentración de hemoglobina corpuscular media, RDW: ancho de distribución eritroide, PLT: recuento de plaquetas, VMP: volumen plaquetario medio, PDW: ancho de distribución plaquetario, PCT: plaquetocrito

\section{DISCUSIÓN}

Los IBR son de gran importancia en la toma de decisiones diagnósticas, terapéuticas y/o de monitoreo. Tener los IBR de la población a la cual se le presta algún servicio es esencial al momento de emitir alguna conclusión, debido a que los parámetros que se miden en el laboratorio clínico suelen variar en relación a las características individuales como la actividad física, las condiciones del entorno y la altura sobre nivel del mar donde reside una población (22-24).

Un estudio realizado por Mantilla et al., encontró que algunos donantes recurrentes pueden disminuir los depósitos de hierro, siendo más frecuente en donantes del sexo femenino, comprometiendo así su estado de salud (25). Para tratar de detectar esta y otras alteraciones, el banco de sangre determina un plazo mínimo de donación, además de una encuesta $y$ mediciones antropométricas como estatura, peso e índice de masa corporal, los cuales dan un estimado de las condiciones generales de salud de la persona. Sin embargo, es posible cursar con deficiencias de hierro e igualmente ser asintomático, por lo que las anteriores medidas son insuficientes para determinar el estado de los depósitos de hierro. La medición de ferritina resuelve este problema, ya que está asociada con los niveles de depósito 
del hierro. Asimismo, la prueba de PCR permite evidenciar alteraciones biológicas en los participantes, debido a que esta es una proteína de fase aguda que se incrementa en la presencia de inflamaciones e infecciones, y puede estar aumentada hasta por 30 días luego de la resolución del evento adverso, minimizando así errores de selección poblacional $(26,27)$.

En el presente estudio se incluyeron donantes que no fueran repetitivos. Según la investigación realizada por Mantilla et al., los donantes repetitivos presentan reservas más bajas de hierro cuya recuperación tarda más tiempo que el indicado en los protocolos para una próxima donación. Por lo tanto, este tipo de donante siempre estará expuesto a la disminución progresiva de las reservas que resulta en una eritropoyesis deficiente en hierro, que provoca anemia (18), lo que demuestra la importancia de un análisis más cuidadoso del estado de este mineral. La ferritina, por ejemplo, es un parámetro válido para ello, por lo cual se decidió implementarla en el presente trabajo.

para el análisis de los resultados de este trabajo no se estratificó por grupo etario los parámetros analizados, debido a que, en una publicación realizada por Pérez et al., concluyeron que los valores de los parámetros hematológicos no varían siognificativamente en muestras poblacionales homogéneas con respecto a las edades de 18 a 55 años (5), las mismas que fueron tomadas en este trabajo. Además, la población del presente estudio no contó con las características etarias para dicha estratificación.

Orrego, M. evaluó los niveles de hemoglobina en deportistas que pertenecen a la ciudad de Medellín, encontró que dependiendo de la cantidad y exigencia de actividad física, dichos parámetros varían: en mujeres de 11,1 hasta 15,6 g/dL $y$, en hombres desde 13,0 hasta $17,9 \mathrm{~g} / \mathrm{dL}$, estos valores difieren a simple vista con respecto a los obtenidos en el presente estudio (mujeres: 12,22 - 15,38 g/dL y hombres: 14,05 - 17,80), por lo tanto es indispensable conocer la actividad física de cada persona antes de realizar el hemograma para correlacionar sus resultados correctamente (28). En un estudio realizado por Donado et al., en el Hospital Pablo Tobón Uribe de la ciudad de Medellín, se encontraron valores de hemoglobina y hematocrito tanto en hombres como en mujeres, similares a los obtenidos en esta investigación: para hombres un promedio de hemoglobina $15,96 \mathrm{~g} / \mathrm{dl}( \pm 1,11) \mathrm{y}$ hematocrito de $46,93 \%( \pm 3,27)$, para mujeres se obtuvo hemoglobina de $14,10 \mathrm{~g} / \mathrm{dl}( \pm 1,00) \mathrm{y}$ hematocrito de $41,64 \%( \pm 2,96)$. Cabe resaltar que ambas poblaciones evaluadas pertenecen a la misma ciudad (29). Se ha encontrado que la concentración de hemoglobina incrementa conforme aumenta la altura sobre el nivel del mar. En Costa Rica se hallaron valores de hemoglobina con una media de 15,58 g/dL para hombres y $13,9 \mathrm{~g} / \mathrm{dL}$ para mujeres, concentraciones que no difieren en gran medida de las de este estudio, debido a que la altura sobre el nivel del mar de Costa Rica, más específicamente de Meseta Central y la ciudad de San José, solo varía un poco menos de 100 metros en comparación con la de Medellín (15).

Las variaciones por sexo en el caso del recuento eritrocitario, el cual fue menor en mujeres que en hombres, se pueden explicar por la presencia de andrógenos en el sexo masculino, que son inductores del sistema eritropoyético, así como los estrógenos son supresores del mismo (30). Se ha descrito que la testosterona estimula la eritropoyesis, pero igualmente inhibe la ventilación pulmonar generando una reducción en la saturación arterial de oxígeno. Sin embargo, lo anterior se ve compensado por un aumento progresivo de eritrocitos y hemoglobina que a su vez se refleja en los cambios de parámetros hemáticos y a la comparación por género (23). El periodo menstrual también puede explicar los rangos menores de hemoglobina, hematocrito y glóbulos rojos en mujeres, debido a la pérdida de sangre en cada ciclo y al tiempo que lleva reponer lo perdido por parte de la médula ósea, esto lleva a un aumento de la eritropoyetina que puede inducir, además de un estímulo en la eritropoyesis, una mayor producción de plaquetas al aumentar su recuento en mujeres $(28,30,31)$. Sin embargo, no se encontraron diferencias sionificativas entre hombres y mujeres para el recuento de plaquetas ( $P=0,338$ ).

Hirokawa et al. publicaron que las altas cargas de estrógenos e interleucinas (IL-6, IL-10) ) en las mujeres logran ampliar la vida media de algunas células como los linfocitos, además de facilitar el recambio de estos, con lo que se explica la diferencia en el recuento leucocitario a expensas de linfocitos encontrados en el presente estudio, donde se hallaron diferencias estadísticamente sionificativas en el recuento de los linfocitos $(p=0,001)$, evidenciado en la diferencia del recuento de leucocitos entre hombres y mujeres (32).

Los intervalos obtenidos con el estadístico (X \pm $Z_{\text {affa/2 }} * \mathrm{DS} / \mathrm{V}_{\mathrm{n}}$ ) por sexo, en este estudio, no difieren con 
los resultados del realizado por Molina et al., en Medellín (5), debido a que las poblaciones evaluadas fueron personas sanas habitantes de la ciudad, y se utilizó el mismo estadístico para calcular los IBR. Cabe aclarar que este estudio usó un equipo hematológico diferente. Esta fórmula genera IBR más precisos, pero en presencia de una muestra poblacional con valores hematológicos homogéneos se obtienen rangos más estrechos de dichos valores, lo que podría dejar por fuera del intervalo, datos que aún se podrían considerar normales según lo revisado en la literatura. Esto llevó a considerar usar la fórmula $\mathrm{X} \pm(\mathrm{DS}$ * 1,96$)$ propuesta por la IFCC, con la que se obtuvieron intervalos más amplios que permitieron incluir datos que presentan una mayor dispersión, pero que aún se consideran valores normales y ofrecen unos IBR más acordes a los establecidos para la población de Medellín, estos IBR fueron los analizados en este trabajo.

\section{CONCLUSIONES}

Los resultados del presente estudio comparados con los realizados en otros países muestran diferencias en la mayoría de los parámetros hematológicos, sustentando la aseveración de que cada laboratorio debe establecer sus respectivos valores de referencia, de acuerdo a la población a la que presta sus servicios.

La diferencia en Ios IBR entre mujeres y hombres obtenidas en este y otros estudios confirma la obligación de establecer valores de referencia adecuados para la población de acuerdo con sus características: el sexo, la edad, altitud de residencia $\mathrm{y}$ actividad física, para así tomar decisiones clínicas más acertadas.

\section{CONFLICTOS DE INTERESES}

Ninguno por declarar.

\section{REFERENCIAS BIBLIOGRÁFICAS}

1. Campuzano Mata G. Del hemograma manual al hemograma de cuarta generación. Medicina \& Laboratorio. 2007;13(11-12):511-50.

2. Vásquez Castillo LF. EI hemograma y su interpretación. Ucimed [Internet]. 2011:1-1. Disponible en: https://7ucimed.files.wordpress.com/2011/08/hemograma-dr-vasquez.pdf

3. Campuzano Maya G. Interpretación del hemograma automatizado: claves para una mejor utilización de la prueba. Medicina \& Laboratorio. 2013;19(1-2):11-68.

4. Mindray. BC-5000. Auto Hematology Analyzer. 2009. Available from: http://www.rapiddiagnostic.in/pdf/ BC-5000.pdf

5. Molina K, Vargas E, Tavera S, Pérez R, Mantilla Y, Cardona J. Intervalos biológicos de referencia del hemograma en personas sanas, Medellín, 2012. Medicina \& Laboratorio. 2013;19(5-6):267-81.

6. Instituto Colombiano de Normas Técnicas y Certificación. Norma Técnica Colombiana NTC-ISO 15189. Bogotá: ICONTEC; 2014. Disponible en: https:// tienda.icontec.org/wp-content/uploads/pdfs/NTCISO15189.pdf

7. Huerta Aragonés JH, Cela de Julián E. Hematología práctica: interpretación del hemograma y de las pruebas de coagulación. En: AEPap, editor. Curso de Actualización Pediatría 2018. Madrid: Lúa; 2018. p. 507-6. Disponible en: https://www.aepap.org/sites/ default/files/507-526_hematologia_practica.pdf

8. Horowitz G, Altaie S, Boyd J, Ceriotti F, Garo U, Horn p et al. Defining, establishing, and verifying reference intervals in the clinical laboratory; approved guideline-third edition. Clinical \& Laboratory Standards Institute [Internet]. 2010;28(30):i-59. Available from: https://clsi.org/media/1421/ep28a3c_sample.pdf

9. Fuentes Arderiu X. Intervalos de referencia biológicos1. NOTICONAQUIC. 2011;54:46-51. Disponible en: http://www.ifcc.org/media/215857/Intervalos\%20 de\%20referencia\%20biol\%C3\%B3gicos\%20DIV.pdf

10. Queralt J, Amoja F, Cortes M, Domenech M, Fuemes J, Llagoslera $M$, et al. Concepto de valores de referencia en Química Clínica. Quím Clín. 1983;2(1):39-41.

11. Sáenz K, Narváez L, Cruz M. Valores de referencia hematológicos en población altoandina ecuatoriana establecidos con el uso del analizador Sysmex XE2100. Rev Mex Patol Clin. 2008;55(4):207-15.

12. Gómez Gerique JA. Proteína C reactiva como marcador de inflamación [Internet]. Santander: Programa de Formación Continuada a Distancia; 2010. Disponible en: https:/www researchgate.net/publication/257924820_Proteina_C_reactiva_como_marcador_de_inflamacion 
13. Coy LS, Castillo M, Mora AI, Oliveros AL, Vélez Z. Estrategias diagnósticas utilizadas para detectar deficiencias de hierro subclínicas y asociadas a enfermedades crónicas. Nova. 2005;3(4):58-68. file://C:/ Users/Home/Downloads/AEFA.FCD2010.T1 qxd\%20 (2).pdf

14. Klever F, Gonzalón S, Narváez L, Cruz M, Checa C. Valores de referencia hematológicos en población afroecuatoriana de Esmeraldas-Ecuador. Rev Fac Cien Med (Quito). 2012;37(1-2):55-64.

15. Sáenz G, Arroyo G, Valenciano E. valores normales de hemoglobina y hematocrito en adultos. Rev Méd Hosp NaI Niños. 1971:6(1):53-70.

16. Torrens $M$. Interpretación clínica del hemograma. RMCLC. 2015;26(6):713-25. DOI 10.1016/j.rmcIc. 2015.11 .001

17. Mantilla Gutiérrez CY, Pérez R, Cardona Arias J. Hierro corporal en donantes habituales de un banco de sangre de Medellín-Colombia. Rev Cubana Hematol Inmunol Hemoter [Internet]. 2014:30(3). Disponible en: http://www.revhematologia.sld.cu/index.php/hih/ article/view/205/129

18. Parés J, Borda N, Santiago S, Benito C, Aranda C. Evaluación de los parámetros de desempeño de un contador hematológico. Acta Bioquím Clín Latinoam. 2015;49(4):399-407.

19. Colombia. Ministro de Salud. Decreto 1571 de 1993 por el cual se reglamenta parcialmente el Título IX de la Ley 09 de 1979. Diario Oficial, 40989 (Agosto 12 de1993).

20. Carey N, Middle J, Durham P, Pierson-Perry J, Hauck W. Smith M, et al. User verification of precision and estimation of bias; approved guideline-third edition [Internet]. United States: Clinical and Laboratory Standards Institute; 2012. Available from: https://clsi. org/media/1431/ep15a3_sample.pdf

21. Isaza Echeverri L, Acevedo Arroyave E, Hernández Barajas F. Comparación de pruebas de normalidad. XXV Simposio de Estadística [Internet]. 2015. Disponible en: http://ciencias.bogota.unal.edu.co/fileadmin/ content/eventos/simposioestadistica/documentos/ memorias/MEMORIAS_2015/Comunicaciones/Est_ Matematica/Isaza_Acevedo__Hernandez_Pruebas_ Normalidad.pdf
22. Colombia. Ministerio de Salud. Resolución 008430. Por la cual se establecen las normas científicas, técnicas y administrativas para la investigación en salud. Bogotá: El Ministerio; 1993.

23. Gonzales G. Hemoglobina y testosterona : importancia en la aclimatación y adaptación a la altura. Rev Perú Med Exp Salud Pública. 2011;28(1):92-100.

24. Roshan TM, Rosline H, Ahmed SA, Rapiaah M, Zaidah AW, Khattak MN. Hematological reference values of healthy malaysian population. Int J Lab Hematol. 2009;31(5):505-12.

25. Mantilla-Gutiérrez CY, Cardona-Arias J. Prevalencia de la deficiencia de hierro en donantes de sangre. Revisión bibliográfica del período 2001-2011. Rev Esp Salud Pública. 2012;86(4):357-69.

26. Orrego M, Pérez M, Pérez M, Cheyre J, Mardones R. Valores plasmáticos de proteína $C$ reactiva $(\mathrm{PCR})$ en cirugía ortopédica electiva. Rev Méd Chile. 2005; 133:1341-8.

27. Aguirre G, Falla A, Sánchez W. Correlación de los marcadores inflamatorios (proteína $C$ reactiva, neutrofilia y leucocitosis) en las diferentes fases de la apendicitis aguda. Rev Colomb Cir. 2014;29:110-5.

28. Orrego ML. Valores de hematocrito y de hemoglobina en deportistas evaluados en Instituto de Deportes de Medellín (Colombia). Act Med Col. 2007;32(4): 196-205.

29. Donado Gómez JH, Ramírez González JA, Trujillo Castro SM, Barco Atehortúa GE, Jaramillo Velásquez $\mathrm{S}$. Valores de hemoglobina y hematocrito en más de 100 mil donantes del banco de sangre del Hospital Pablo Tobón Uribe, Medellín-Colombia (1538 msnm). Medicina UPB. 2013;32(2):138-43.

30. Lichtman M. Williams manual de hematología. $8^{\mathrm{a}}$ ed. México: McGraw Hill. 2014.

31. Skikne BS, Flowers CH, Cook JD. Serum transferrin receptor: a quantitative measure of tissue iron deficiency. Blood. 1990 May;75(9):1870-6.

32. Hirokawa K, Utsuyama M, Hayashi Y, Kitagawa M, Makinodan T, Fulop T. Slower immune system aging in women versus men in the Japanese population. Immun Ageing. 2013 May;10(1):19. DOI 10.1186/17424933-10-19. 